\title{
Phase-Locking and Coherent Power Combining of Linearly Chirped Optical Waves
}

\author{
Naresh Satyan, ${ }^{1, *}$ Arseny Vasilyev, ${ }^{1}$ George Rakuljic, ${ }^{2}$ Jeffrey O. White, ${ }^{3}$ and Amnon \\ Yariv $^{1}$ \\ ${ }^{1}$ Department of Applied Physics and Materials Science, California Institute of Technology, \\ 1200 E. California Blvd. 136-93, Pasadena, CA 91125, USA \\ ${ }^{2}$ Telaris Inc., 2118 Wilshire Blvd. \#238, Santa Monica, CA 90403, USA \\ ${ }^{3}$ Army Research Laboratory, 2800 Powder Mill Road, Adelphi, MD 20783, USA \\ *Corresponding author: naresh@caltech.edu
}

\begin{abstract}
Rapidly chirped optical waveforms reduce stimulated Brillouin scattering in fiber amplifiers, enabling higher output powers. This work demonstrates homodyne and heterodyne phase-locking, and coherent combining, of linearly chirped optical waves using acoustooptic frequency shifters.

(C) 2011 Optical Society of America

OCIS codes: (140.3298) Laser beam combining; (140.3600) Lasers, tunable.
\end{abstract}

Laser power combining has been an active research area for the past few years, aimed at achieving optical powers larger than the output powers of individual elements. In particular, coherent beam combining of fiber amplifiers using active feedback is one of the most promising approaches towards realizing very large output powers [1]. The output power of a single fiber amplifier is typically limited by stimulated Brillouin scattering (SBS) in the fiber, and spectral modulation of the seed laser to several nanometers is typically used to increase the available output power. Such broadband spectral modulation, however, requires precise path-length matching for coherent combining $[2,3]$.

In a recent collaborative effort, we have demonstrated that the use of a rapid broadband swept-frequency laser (SFL) produces a significant enhancement in the threshold power for SBS [4] in optical fibers. The SFL incorporates optoelectronic feedback that ensures that its output frequency chirp is precisely linear [5]. In this work, we demonstrate the phase-locking of separate optical paths seeded by a common SFL master oscillator. Taking advantage of the high linearity of the optical chirp, we use an acoustooptic frequency shifter (AOFS) to electronically compensate for differences and fluctuations in optical path lengths. This combined approach for SBS suppression and coherent combining therefore enables higher output powers than the coherent combining of single-frequency fiber amplifiers.

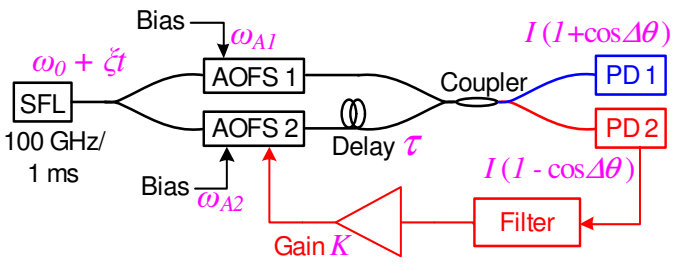

(a)

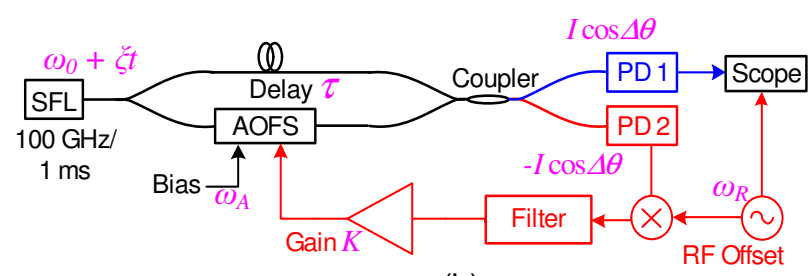

(b)

Fig. 1. (a) Homodyne and (b) heterodyne phase-locking architectures. $\Delta \theta$ is the phase difference between the two combining arms. PD: Photodetector.

The schematic for the experimental demonstration of phase-locking of chirped optical waves in the homodyne and heterodyne configurations is shown in Fig. 1. The input source is a DFB semiconductor laser-based precisely linear SFL with a chirp slope $\xi$ of $100 \mathrm{GHz}$ in $1 \mathrm{~ms}$ [5]. The setup is similar to that of earlier work using AOFSs for phase correction [2], with the critical distinction that the seed source is swept coherently over a very large bandwidth. The key insight is that the precise linearity of the chirped laser means that a differential path delay $\tau$ translates into a relative frequency shift $\xi \tau$ between the combining arms, which can be corrected using an AOFS. A fiber path length mismatch of $2 \mathrm{~m}$ at a chirp rate of $10^{14} \mathrm{~Hz} / \mathrm{s}$ can be electronically compensated by a frequency shift of $1 \mathrm{MHz}$. The control loop eliminates phase errors due to environmental fluctuations, at frequencies within the loop bandwidth. 
The steady state solutions for the two loops in Fig. 1 are

$$
\begin{aligned}
\Delta \theta_{\text {Homodyne }} & =\cos ^{-1}(1+\Delta \omega / K), \\
\Delta \theta_{\text {Heterodyne }} & =\omega_{R} t+\sin ^{-1}(\Delta \omega / K),
\end{aligned}
$$

where the free-running frequency difference $\Delta \omega$ is given by $\left(\omega_{A 2}-\omega_{A 1}-\xi \tau\right)$ and $\left(\omega_{R}-\omega_{A}-\xi \tau\right)$ for the homodyne and heterodyne cases respectively, and $K$ is the loop gain. From Eq. (1), the phase difference between the two optical waves can be controlled by varying the bias frequency of the AOFS, and hence $\Delta \omega$.

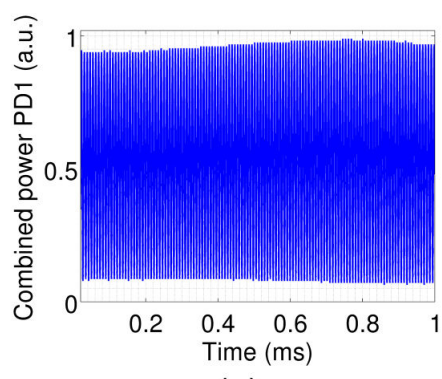

(a)

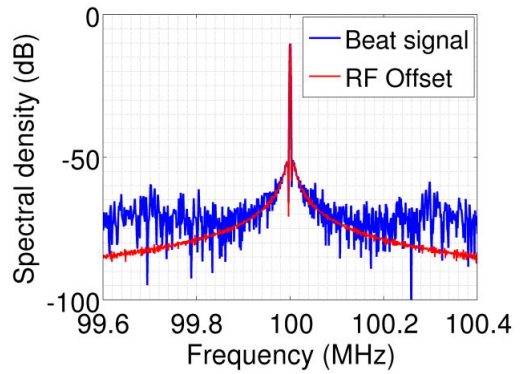

(c)

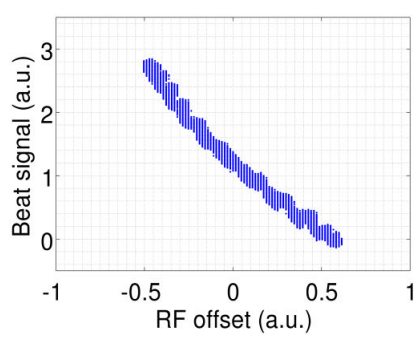

(d)

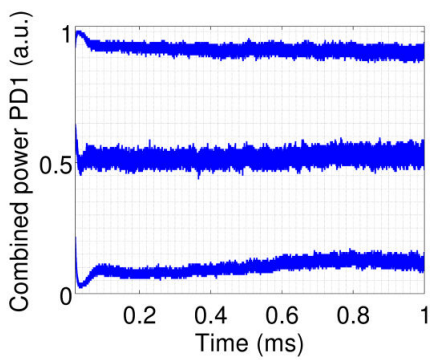

(b)

Fig. 2. Experimental results. Homodyne combining: (a) unlocked, and (b) phase-locked with three different values of $\Delta \omega$. Heterodyne locking: (c) Spectra of the optical beat signal and the RF offset, and (d), (e) Lissajous figures of the optical beat signal and the RF offset, for different values of $\Delta \omega$.

The homodyne and heterodyne phase-locked loops of Fig. 1 were constructed using polarization maintaining fiber components, AOFSs with a nominal frequency shift of $100 \mathrm{MHz}$, and lag filters. The RF offset frequency in the heterodyne loop was $100 \mathrm{MHz}$. The loop bandwidth obtained was about $\sim 70 \mathrm{kHz}$, limited by the driver for the AOFS. The experimental results are shown in Fig. 2. The measurements were performed over the duration of the chirp (1 ms). Transient effects during the beginning of the chirp are not shown. By varying $\Delta \omega$, control of the relative phase between the combined arms was demonstrated.

The authors acknowledge the support of DARPA (MTO), and the High Energy Laser Joint Technology Office.

\section{References}

1. S. J. Augst, J. K. Ranka, T. Y. Fan, and A. Sanchez, "Beam combining of ytterbium fiber amplifiers (invited)," J. Opt. Soc. Am. B 24, 1707-1715 (2007).

2. S. J. Augst, T. Y. Fan, and A. Sanchez, "Coherent beam combining and phase noise measurements of ytterbium fiber amplifiers," Opt. Lett. 29, 474-476 (2004).

3. G. D. Goodno, S. J. McNaught, J. E. Rothenberg, T. S. McComb, P. A. Thielen, M. G. Wickham, and M. E. Weber, "Active phase and polarization locking of a $1.4 \mathrm{~kW}$ fiber amplifier," Opt. Lett. 35, 1542-1544 (2010).

4. J. White, O. Okusaga, J. Cahill, C. Mungan, G. Rakuljic, A. Vasilyev, N. Satyan, and A. Yariv, "Suppression of stimulated Brillouin scattering in optical fibers using broadband frequency chirps," Manuscript in preparation.

5. N. Satyan, A. Vasilyev, G. Rakuljic, V. Leyva, and A. Yariv, "Precise control of broadband frequency chirps using optoelectronic feedback," Opt. Express 17, 15,991-15,999 (2009). 